\title{
Dynamic kinetic energy potential for orbital-free density functional theory
}

\author{
Daniel Neuhauser, ${ }^{1, \text { a) }}$ Shlomo Pistinner, ${ }^{1}$ Arunima Coomar, ${ }^{1}$ Xu Zhang, ${ }^{2}$ and Gang Lu ${ }^{2}$ \\ ${ }^{1}$ Department of Chemistry and Biochemistry, UCLA, Los Angeles, California 90095-1569, USA \\ ${ }^{2}$ Department of Physics and Astronomy, California State University, Northridge, California 91330-8268, USA
}

(Received 9 January 2011; accepted 15 March 2011; published online 8 April 2011)

A dynamic kinetic energy potential (DKEP) is developed for time-dependent orbital-free (TDOF) density function theory applications. This potential is constructed to affect only the dynamical $(\omega \neq 0)$ response of an orbital-free electronic system. It aims at making the orbital-free simulation respond in the same way as that of a noninteracting homogenous electron gas (HEG), as required by a correct kinetic energy, therefore enabling extension of the success of orbital-free density functional theory in the static case (e.g., for embedding and description of processes in bulk materials) to dynamic processes. The potential is constructed by expansions of terms, each of which necessitates only simple time evolution (concurrent with the TDOF evolution) and a spatial convolution at each time-step. With 14 such terms a good fit is obtained to the response of the HEG at a large range of frequencies, wavevectors, and densities. The method is demonstrated for simple jellium spheres, approximating $\mathrm{Na}_{9}{ }^{+}$and $\mathrm{Na}_{65}{ }^{+}$clusters. It is applicable both to small and large (even ultralarge) excitations and the results converge (i.e., do not blow up) as a function of time. An extension to iterative frequency-resolved extraction is briefly outlined, as well as possibly numerically simpler expansions. The approach could also be extended to fit, instead of the HEG susceptibility, either an experimental susceptibility or a theoretically derived one for a non-HEG system. The DKEP potential should be a powerful tool for embedding a dynamical system described by a more accurate method (such as time-dependent density functional theory, TDDFT) in a large background described by TDOF with a DKEP potential. The type of expansions used and envisioned should be useful for other approaches, such as memory functionals in TDDFT. Finally, an appendix details the formal connection between TDOF and TDDFT. @ 2011 American Institute of Physics. [doi:10.1063/1.3574347]

\section{INTRODUCTION}

The orbital-free (OF) description of quantum electronic systems dates back to the work of Thomas and Fermi, von Weizsäcker, Dirac, Slater, and eventually Kohn and Hohenberg; the work of the latter laid the theoretical foundation both to density functional theory (DFT) and also to orbitalfree DFT (OFDFT). However, the work of Kohn and Sham shifted interest away from OFDFT to orbital-based DFT or later its equivalent, density-matrix DFT, due to the improved description of the kinetic energy. (For a review, see Ref. 1.) Nevertheless, the last two decades have witnessed a flurry of interest in OFDFT; as computer power grows, larger and larger systems can be simulated by OFDFT thanks to its favorable linear scaling of computational time with respect to the size of the electronic system. ${ }^{2-17}$ In particular, OFDFT is indispensable for embedding approaches, where OFDFT is used to describe both the large surrounding environment as well as the interaction between the environment and a smaller active region. ${ }^{18-21}$

Most of the research activity in orbital-free DFT has been devoted to static properties. Specifically, appropriate kinetic energy functionals (KEFs) have been generated by fitting to the response of static electronic systems. This approach has led to development of accurate KEF for both metals and semi-

\footnotetext{
a)Electronic mail: dxn@chem.ucla.edu.
}

conductors. Although most of the KEFs have been used for simulating static properties, progress has also been made for describing excitonic coupling. However, up to date, all present OFDFT approaches are based on the static description of the KEF.

Time-dependent orbital-free (TDOF) descriptions have also been explored. ${ }^{22-27}$ The work of Runge and Gross on time-dependent DFT (TDDFT) lays the formal foundations for $\mathrm{TDOF},{ }^{28}$ and there have been practical simulations which use the static OFDFT potential in studying metal and fullerene clusters. ${ }^{29}$ Formally it is possible to relate the TDDFT and TDOF potentials; this can be done directly, as shown in Appendix.

Moving away from the formal correspondence to a practical TDOF simulation, here we propose to improve upon TDOF by adding a potential which imposes the correct dynamical susceptibility. Our proposal is similar in spirit to the addition of the static linear response term in modern OFDFT theories, except our aim is dynamic response. We introduce therefore a new quantity, which we label dynamic kinetic energy potential (DKEP). The DKEP is a memory potential, but it is different from the usual memory potentials in TDDFT, as its aim is to capture only the kinetic energy. As we show, the DKEP is numerically feasible so it could become a general tool for OF studies. The implications of DKEP could be widely ranging, as it should allow for smooth embedding in general dynamical problems. In fact, any place where the 
background can respond, one should employ DKEP and not resort to the static response; such applications could also be excitonic coupling through a medium described by OF approaches; or even the interaction of high energy electrons with metals.

Another possibly relevant case for OFDFT, even by its own (i.e., separate from embedding issues), is Maxwell FDTD (finite-difference time domain) simulations; here, the use of OFDFT could potentially be sufficiently feasible for nanometric systems, while at the same time allow for nonlocal response in a way not afforded by standard treatments ${ }^{30}$ of metals in FDTD.

The remainder of the paper is organized as follows. In Sec. II we discuss the transformation of TDDFT to TDOF, and in Sec. III we introduce the DKEP. The fitting procedure is presented in Sec. IV. Simulations of model systems are discussed in Sec. V. Implications and possible extensions are discussed in Sec. VI, including the plausible type of susceptibility to be used, frequency versus time-based applications and propagation, implications for embedding, and future improvements to the numerical representation. Conclusions follow in Sec. VII. Appendix covers the relation between TDDFT and TDOF.

\section{DKEP: A POTENTIAL FOR TRANFROMING THE NONINTERACTING N-ELECTRON PROBLEM INTO A SINGLE-PARTICLE PROBLEM}

Assume we have a system of electrons in $N$ electrons in $N$ time-dependent orbitals, $\phi_{j}(\mathbf{r}, t)$ which formally should be propagated under a TDDFT potential ${ }^{28}$

$i \frac{\partial \phi_{j}(\mathbf{r}, t)}{\partial t}=-\frac{\Delta \phi_{j}(\mathbf{r}, t)}{2 m}+\left(v_{\mathrm{TDDFT}}(\mathbf{r}, t)+v_{\mathrm{ext}}(\mathbf{r}, t)-\mu\right) \phi_{j}$,

where we introduced the chemical potential, and $\nu_{\text {TDDFT }}$ includes the interaction with the background nuclear density $\rho_{N}$, the direct electron-electron repulsion, and any desired exchange-correlation potential (possibly including a memory functional contribution):

$v_{\mathrm{TDDFT}}(\mathbf{r}, t)=\int \frac{\rho\left(\mathbf{r}^{\prime}, t\right)-\rho_{N}\left(\mathbf{r}^{\prime}, t\right)}{\left|\mathbf{r}-\mathbf{r}^{\prime}\right|} d^{3} \mathbf{r}^{\prime}+v_{\mathrm{TD}-\mathrm{XC}}[\rho](\mathbf{r}, t)$.

In addition, $v_{\text {ext }}$ is any desired perturbation (typically interaction with an electromagnetic field).

The TDOF approach tries to circumvent the full TDDFT propagation of the $N$ orbitals, and uses instead a single-wavefunction-like orbital $\Psi$ (labeled the Madelung wavefunction $^{31}$ ) which is related to the time-dependent density:

$$
\Psi(\mathbf{r}, t)=\sqrt{\rho(\mathbf{r}, t)} e^{i \chi(\mathbf{r}, t)},
$$

and is normalized to the total number of electrons,

$$
\langle\Psi \mid \Psi\rangle=\int \rho(\mathbf{r}, t) d \mathbf{r}=N .
$$

(Note that arguably the method should be better labeled as "time-dependent single-orbital" rather than "time-dependent orbital-free," but we will use the prevailing notation.) The goal of TDOF is to determine the proper extra potential, $v_{\mathrm{OF}}$, which will allow to determine $\Psi$ from a Schrödinger-like equation:

$$
i \frac{\partial \Psi}{\partial t}=-\frac{\Delta \Psi}{2 m}+(v(\mathbf{r}, \rho, t)-\mu) \Psi,
$$

where the total potential is now

$$
v=v_{\mathrm{OF}}+v_{\mathrm{TDDFT}}+v_{\mathrm{ext}} .
$$

Put differently, in addition to the TDDFT potential we introduce an additional term, $\nu_{\mathrm{OF}}$, the goal of which is to ensure that the density from the orbital-free approach is the same as that from the many-orbital TDDFT propagation. The DKEP, $v_{\mathrm{OF}}$ is (together with the single-particle kinetic energy) essentially the orbital-free approximation to the kinetic energy, i.e., the potential which allows that conversion of the N-orbital propagation into a single-orbital dynamics.

A formal construction of the DKEP when the TDDFT problem is solved is represented in Appendix; this is useful for connecting to the TDDFT formulation. In practice, of course, the TDDFT orbitals will not be known in a purely TDOF simulation, so we instead concentrate in the main body of the paper on a fit of the DKEP.

We write the DKEP potential as having three terms: Thomas-Fermi, von Weizsäcker, and a correcting term discussed later:

$$
v_{\mathrm{OF}}=\frac{\left(3 \pi^{2}\right)^{\frac{2}{3}}}{2 m} \rho^{\frac{2}{3}}-(a-1) \frac{\Delta|\Psi|}{2 m|\Psi|}+v_{\mathrm{OF}}^{\prime},
$$

where $a$ is the numerical coefficient of the von Weizsäcker term (typically in the range of 1 to 1/9). Note that the shift of the von Weizsäcker term from $a$ to $a-1$ is due to the time dependence in the equation for $\Psi$, as this modification negates the appropriate part of the spatial derivative in the Laplacian term in Eq. (5).

The correcting term is further decomposed of a nonlocal kinetic energy potential $v_{s}$ (i.e., depending on the instantaneous density) as well as an important dynamical term $u$ :

$$
v_{\mathrm{OF}}^{\prime}=v_{s}+u \text {. }
$$

Here $v_{s}$ represents modern kernels for the static orbital-free DFT which depends only on the instantaneous density:

$$
v_{S}(\mathrm{r})=\frac{\delta}{\delta \rho(\mathrm{r}, t)} V_{S},
$$

typically of the form

$$
V_{S}=\int \rho^{\alpha}\left(\mathrm{r}^{\prime}, t\right) Q\left(\rho\left(\mathrm{r}^{\prime}, t\right), \rho\left(\mathrm{r}^{\prime \prime}, t\right), \mathrm{r}^{\prime}, \mathrm{r}^{\prime \prime}\right) \rho^{\beta}\left(\mathrm{r}^{\prime \prime}, t\right) d \mathrm{r}^{\prime} d \mathrm{r}^{\prime \prime},
$$

where $Q$ is a specific material-dependent kernel.

The final term $u$ is the one proposed in this paper; its purpose is to ensure the proper frequency-dependent linear response. Therefore we label it as DKEP, which is explored below.

\section{ORBITAL-FREE SUCSEPTIBILITY AND DKEP}

First, we focus on the simplest possible formulation which is to obtain the DKEP by matching the response of a 
noninteracting single-orbital system to that of a noninteracting homogenous electron gas (HEG). 22, 32,33

We start with a homogenous electron gas with an initial electron density $\rho_{0}$. The unperturbed Madelung wavefunction can be written as:

$$
\Psi_{0}(\mathrm{r}, t)=e^{-i E_{0} t} \sqrt{\rho_{0}},
$$

where

$$
E_{0}=v_{\mathrm{OF}}\left(\rho_{0}\right)-\mu .
$$

The external linear perturbation is of the form

$$
V_{\mathrm{ext}}=\delta V_{\mathrm{ext}}=\eta \exp (i \mathbf{q} \bullet \mathbf{r}-i \omega t)+c . c,
$$

where $\eta$ is the (complex) linear perturbation strength. The response of the Madelung wavefunction is thus

$$
\Psi=\Psi_{0}+e^{-i E_{0} t} \delta \Psi
$$

where $\delta \Psi$ and the response of the other quantities to the perturbation can be written as:

$$
\begin{aligned}
& \delta \Psi=\eta \psi^{+}\left(q, \omega, \rho_{0}\right) e^{i \mathbf{q} \bullet \mathbf{r}-i \omega t}+\eta^{*} \psi^{-*}\left(q, \omega, \rho_{0}\right) e^{i \mathbf{q} \bullet \mathbf{r}-i \omega t} \\
& \delta \rho=\eta \chi_{0} e^{i \mathbf{q} \bullet \mathbf{r}-i \omega t}+c . c . \\
& \delta v_{\mathrm{OF}}=\eta \zeta \chi_{0} e^{i \mathbf{q} \bullet \mathbf{r}-i \omega t}+c . c . \\
& \delta v_{s}=\eta \sigma\left(q, \rho_{0}\right) \chi_{0} e^{i \mathbf{q} \bullet \mathbf{r}-i \omega t}+c . c . \\
& \delta u=\eta \zeta\left(q, \omega, \rho_{0}\right) \chi_{0} e^{i \mathbf{q} \bullet \mathbf{r}-i \omega t}+c . c .
\end{aligned}
$$

where these quantities have the obvious meaning

$$
\zeta=\frac{\delta v_{\mathrm{OF}}}{\delta \rho}, \sigma=\frac{\delta v_{s}}{\delta \rho}, \xi=\frac{\delta u}{\delta \rho} .
$$

Note that as usual in linear response for wavefunctions, the Madelung wavefunction contains both phases of the perturbation but not with the same strength. Also, here the noninteracting susceptibility, $\chi_{0}\left(\rho_{0}, q, \omega\right)$, is the response of the density to the external potential in the absence of an electronelectron interaction. Finally, the instantaneous potential is independent of the frequency.

To derive $\chi_{0}$ for TDOF, we first note that since the wavefunction is related to the density,

$$
\delta \rho=\Psi^{*} \delta \Psi+c . c,
$$

it follows that

$$
\chi_{0}=\sqrt{\rho_{0}}\left(\psi^{+}+\psi^{-}\right) .
$$

The time-dependent equation for the Madelung wavefunction,

$$
i \frac{d}{d t} \delta \Psi=\frac{q^{2}}{2 m} \delta \Psi+\left(\delta v_{\mathrm{OF}}+\delta v_{\mathrm{ext}}\right) \Psi_{0},
$$

yields then

$$
\begin{aligned}
\omega \psi^{+} & =\frac{q^{2}}{2 m} \psi^{+}+\sqrt{\rho_{0}}\left(\zeta \chi_{0}+1\right) \\
-\omega \psi^{-} & =\frac{q^{2}}{2 m} \psi^{-}+\sqrt{\rho_{0}}\left(\zeta \chi_{0}+1\right),
\end{aligned}
$$

i.e., by combining with Eq. (18),

$$
\chi_{0}=\rho_{0}\left(1+\zeta \chi_{0}\right)\left(\frac{1}{\omega-\frac{q^{2}}{2 m}}-\frac{1}{\omega+\frac{q^{2}}{2 m}}\right),
$$

which rearranges to yield

$$
\frac{1}{\chi_{0}\left(q, \omega, \rho_{0}\right)}=\frac{1}{\rho_{0}\left(\frac{1}{\omega-\frac{q^{2}}{2 m}}-\frac{1}{\omega+\frac{q^{2}}{2 m}}\right)}-\zeta\left(q, \omega, \rho_{0}\right)
$$

Next, we notice that from Eqs. (7) and (15) the response of the kinetic energy part has the form:

$$
\zeta=\frac{\left(3 \pi^{2}\right)^{\frac{2}{3}}}{3 m} \rho_{0}^{-\frac{1}{3}}+(a-1) \frac{q^{2}}{4 m \rho_{0}}+\sigma+\xi .
$$

There are two possibilities now for fitting, which will lead to different results. In both cases, however, the role for $\xi$ is clear: it needs to match the orbital-free susceptibility $\chi_{0}$ to the desired susceptibility, as explained below.

The first is to note that in Eq. (23) the only frequencydependent part is the last one, $\xi\left(q, \omega, \rho_{0}\right)$. We can then apply Eq. (22) at a fixed frequency, $\omega_{0}$, which typically could have a zero real part and a small imaginary part to ensure nonsingularity. By subtracting the susceptibilities at a general and fixed frequency (i.e., $\omega$ and $\omega_{0}$ ), we remove the contribution of the instantaneous terms (Thomas-Fermi, von Weizsäcker, and the static part), yielding the following expression:

$$
\begin{aligned}
\xi\left(q, \omega, \rho_{0}\right)= & \frac{1}{\rho_{0}\left(\frac{1}{\omega-\frac{q^{2}}{2 m}}-\frac{1}{\omega+\frac{q^{2}}{2 m}}\right)}+\frac{q^{2}}{4 m \rho_{0}}-\frac{1}{\chi_{0}\left(q, \omega, \rho_{0}\right)} \\
& +\frac{1}{\chi_{0}\left(q, \omega_{0} \rightarrow 0, \rho_{0}\right)} .
\end{aligned}
$$

This choice is ostensibly very simple and yields a welldefined prescription for $u$ which can then be fitted as explained later. The attractive feature of this equation is that it is independent of the specific terms being used such as ThomasFermi, von Weizsäcker, etc. However, it relies on the assumption that Eq. (22) is valid at $\omega_{0}$, i.e., that a successful static fitting has to be achieved. This may or may not be the case in practice. Furthermore, even in case where formally we impose the correct susceptibility at zero frequency, in practice the imposition may not be sufficiently accurate.

We therefore need to consider a second choice, where we impose Eq. (22) only in a specific frequency range and not necessarily for the static case:

$$
\begin{aligned}
\chi_{0}\left(q, \omega, \rho_{0}\right)= & {\left[\left(\frac{\rho_{0}}{\omega-\frac{q^{2}}{2 m}}-\frac{\rho_{0}}{\omega+\frac{q^{2}}{2 m}}\right)^{-1}-\frac{\left(3 \pi^{2}\right)^{\frac{2}{3}}}{3 m} \rho_{0}^{-\frac{1}{3}}\right.} \\
& \left.-(a-1) \frac{q^{2}}{4 m \rho_{0}}-\sigma\left(q, \rho_{0}\right)-\xi\left(q, \omega, \rho_{0}\right)\right]^{-1}
\end{aligned}
$$




\section{FITTING AND APPLYING THE DKEP}

\section{A. DKEP representation}

We will write the dynamic term in a way which manifestly vanishes for static simulations

$$
u(\mathbf{r}, t)=\frac{d y(\mathbf{r}, t)}{d t} .
$$

The goal is then to fit $y$, which we call a generating potential. There are several options for representing the DKEP potential.

One option in static simulations is to expand in the terms of the density around a reference density and integrate analytically over $q \cdot{ }^{18,34}$ Here, we could have taken a similar route but the frequency dependence will still need to be accounted for, i.e., without an explicit integration from time to frequency, as we only have information about previous times during the propagation. We note that if results are required at a single frequency only, then a procedure analogous to the static case (i.e., numerically exact using a reference density) could have been employed. This may be applied in future, more specialized, simulations.

For general time-dependent simulations covering a range of frequencies, we therefore adopt instead a global-fit approach, and represent the 3D parameter-space of wavevectors, frequencies, and densities without a reference density:

$$
\begin{aligned}
y(\mathbf{r}, t)= & \sum_{j} d_{j} \rho(\mathbf{r}, t)^{a_{j}} \int e^{-\kappa_{j}\left|\mathbf{r}-\mathbf{r}^{\prime}\right|-i \omega_{j}\left(t-t^{\prime}\right)} \\
& \times \rho\left(\mathbf{r}^{\prime}, t^{\prime}\right)^{\beta_{j}} d \mathbf{r}^{\prime} d t^{\prime}+c . c,
\end{aligned}
$$

where we chose a large number of terms " $j$ " (typically 14). This expansion is clearly analogous to the static fit.
Here, $d_{j}, \kappa_{j}, \omega_{j}$ are complex parameters but for simplicity we choose $\alpha_{j}, \beta_{j}$ as real (although a complex choice is equally feasible). The real part of $\kappa_{j}$ needs to be positive, and the imaginary part of $\omega_{j}$ is negative.

Next we review the determining equations for the coefficients, and then the practical application of $y$ in the timedomain.

\section{B. DKEP parameters}

In linear response, $\rho(\mathbf{r}, t)=\rho_{0}+\delta \rho$, where $\delta \rho$ is the periodic perturbation of Eq. (15), the generating potential is

$$
y=y_{0}+\delta y,
$$

where $y_{0}=y\left(\rho_{0}\right)$ and

$$
\begin{aligned}
\delta y(\mathbf{r}, t)= & \int \sum_{j} d_{j} \alpha_{j} \rho_{0}^{\alpha_{j}-1} \delta \rho(\mathbf{r}, t) \exp \left(-\kappa_{j}\left|\mathbf{r}-\mathbf{r}^{\prime}\right|\right. \\
& \left.-i \omega_{j}\left(t-t^{\prime}\right)\right) \rho_{0}^{\beta_{j}} d \mathbf{r}^{\prime} d t^{\prime} \\
& +\int \sum_{j} d_{j} \beta_{j} \rho_{0}^{\alpha_{j}} \exp \left(-\kappa_{j}\left|\mathbf{r}-\mathbf{r}^{\prime}\right|\right. \\
& \left.-i \omega_{j}\left(t-t^{\prime}\right)\right) \delta \rho\left(\mathbf{r}^{\prime}, t^{\prime}\right) \rho_{0}^{\beta_{j}-1} d \mathbf{r}^{\prime} d t^{\prime}+c . c .
\end{aligned}
$$

For a periodic perturbation, Eqs. (13) and (15) yield

$$
\delta y=i \eta \chi \frac{\xi}{\omega} e^{i \mathbf{q} \bullet \mathbf{r}-i \omega t}+c . c .
$$
yield

After some straightforward algebra, Eqs. (29) and (30)

\section{DKEP fitting}

$$
\begin{aligned}
\xi\left(q, \omega, \rho_{0}\right)= & -\omega \sum_{j} d_{j} \rho_{0}^{\alpha_{j}+\beta_{j}-1}\left(\frac{8 \pi \alpha_{j}}{\omega_{j} \kappa_{j}^{3}}+\frac{2 i \pi \beta_{j}}{\left(\omega_{j}-\omega\right) q}\left(\frac{1}{(\kappa+i q)^{2}}-\frac{1}{(\kappa-i q)^{2}}\right)\right) \\
& +\omega \sum_{j} d_{j}^{*} \rho_{0}^{\alpha_{j}+\beta_{j}-1}\left(\frac{8 \pi \alpha_{j}^{*}}{\omega_{j}^{*} \kappa_{j}^{* 3}}+\frac{2 i \pi \beta_{j}^{*}}{\left(\omega_{j}^{*}+\omega\right) q}\left(\frac{1}{\left(\kappa^{*}+i q\right)^{2}}-\frac{1}{\left(\kappa^{*}-i q\right)^{2}}\right)\right) .
\end{aligned}
$$

and the Fermi momentum is

$$
k_{F}=\left(3 \pi^{2} \rho_{0}\right)^{1 / 3} .
$$

The fitting is carried out by minimizing an objective function. Specifically, we separate the parameters to the linear coefficients and the set of nonlinear parameters

$$
\mathbf{b} \equiv\left\{\alpha_{j}, \beta_{j}, \operatorname{Re}\left(\kappa_{j}\right), \operatorname{Im}\left(\kappa_{j}\right), \operatorname{Re}\left(\omega_{j}\right), \operatorname{Im}\left(\omega_{j}\right)\right\},
$$


and then define an objective function

$$
\begin{aligned}
J(\mathbf{b}, \mathbf{d})= & \sum_{m} \mid \chi^{\mathrm{HEG}}\left(q_{m}, \omega_{m}, \rho_{m}\right) \\
& -\left.\chi_{0}\left(q_{m}, \omega_{m}, \rho_{m} ; \mathbf{b}\right)\right|^{2}+c|\mathbf{d}|^{2},
\end{aligned}
$$

where the number of terms $m$ is typically $\sim 5000$, and $q_{m}, \omega_{m}, \rho_{m}$ are randomly chosen points within a given range of wavevectors, frequencies, and densities, respectively; the regularization parameter $c$ ensures that the coefficients will not be too large due to over-completeness.

For each choice of the nonlinear parameters, b, we optimize with respect to the linear coefficients, d, i.e., we first find numerically

$$
I(\mathbf{b})=\min _{\mathbf{d}} J(\mathbf{d}, \mathbf{b}) .
$$

Then, we use steepest to optimize the nonlinear parameters, b. Finally, each parameter in the fit has an allowed region with which it varies, and this is accomplished by transforming the parameters; for example, instead of using $\alpha_{j}$ we optimize a related parameter, which is unrestricted and yields $\alpha_{j}$ at a restricted range, and similarly for the other parameters.

In practice, the summation of the terms in $y$ should be viewed as an expansion, rather than a fit; owing to the large number of terms, we find that we can fit well the linear coefficients $d_{j}$ for any reasonable choice of the other nonlinear parameters.

A final technical point is the sampling set of $\left(q_{m}, \omega_{m}, \rho_{m}\right)$. These are chosen randomly within a given desired parameter space. The results depend of course on the sampling space (i.e., the range of values of the wavevector, frequency, and density). However, within a given sampling domain, we found that the results are sufficiently independent of the specific set of sampling points used. For example, to converge well with about $\sim 5000$ randomly chosen sampling points, it is preferable to add a small imaginary part to the frequency, i.e., the sampled frequencies have the form

$$
\omega_{m}=\omega_{r, m}+i \Delta
$$

where in our simulations $\Delta=0.001$ a.u.was sufficient. (The positive imaginary sign is required by the Lindhard expression.) Note that the complex frequency is used both in the Lindhard expression and in Eqs. (25) and (31).

The results of a specific fitting used are presented in Sec. V.

\section{DKEP application}

Next we turn to the application of the DKEP, as part of the time-dependent Schrödinger equation [Eq. (5)]. We represent the DKEP generating potential, $y$, as a standard convolution term

$$
y(\mathbf{r}, t)=\sum_{j} d_{j} \rho(\mathbf{r}, t)^{a_{j}} Q_{j}(\mathbf{r}, t),
$$

where

$$
Q_{j}(\mathbf{r}, t) \equiv \int \exp \left(-\kappa_{j}\left|\mathbf{r}-\mathbf{r}^{\prime}\right|\right) P_{j}\left(\mathbf{r}^{\prime}, t\right) d \mathbf{r}^{\prime},
$$

and

$$
P_{j}(\mathbf{r}, t) \equiv \int_{t^{\prime}=-\infty}^{t^{\prime}=t} \exp \left(-i \omega_{j}\left(t-t^{\prime}\right)\right) \rho\left(\mathbf{r}, t^{\prime}\right)^{\beta_{j}} d t^{\prime},
$$

which is determined by propagating:

$$
\frac{\partial P_{j}(\mathbf{r}, t)}{\partial t}=-i \omega_{j} P_{j}(\mathbf{r}, t)+\rho(\mathbf{r}, t)^{\beta_{j}} .
$$

The initial conditions are that the time derivative of the $P_{j}$ terms vanish (before the application of any perturbation),

$$
\frac{\partial P_{j}\left(\mathbf{r}, t=0^{-}\right)}{\partial t}=0
$$

so that

$$
P_{j 0} \equiv P_{j}(\mathbf{r}, t=0)=-\frac{i \rho_{0}^{\beta_{j}}}{\omega_{j}}
$$

At each time-step, once these $P_{j}(\mathbf{r}, t)$ terms are known, the resulting $Q_{j}(\mathbf{r}, t)$ [Eq. (41)] are determined by the standard spatial convolution with FFT, as it is easy to show that [analogous to Eq. (31)]

$$
Q_{j}(\mathbf{k}, t)=\frac{2 i \pi}{k}\left(\frac{1}{\left(\kappa_{j}+i k\right)^{2}}-\frac{1}{\left(\kappa_{j}-i k_{j}\right)^{2}}\right) P_{j}(\mathbf{k}, t) .
$$

These convolutions are the most expensive part in the applications of the OFDFT term. An alternative is presented later.

There is some flexibility on the choice of how to propagate the time-dependent equation, Eq. (46), and the Madelung equation, Eq. (5). Here, at each time $t$ we did the following:

- First, propagate Eqs. (5) and (46) (for $\left.d \Psi(\mathbf{r}, t) / d t, d P_{j}(\mathbf{r}, t) / d t\right)$, but without including the DKEP in the time-dependent Hamiltonian of Eq. (5); schematically

$$
\Psi(\mathbf{r}, t), P_{j}(\mathbf{r}, t) \rightarrow \Psi^{\prime}(\mathbf{r}, t+d t), P_{j}(\mathbf{r}, t+d t) .
$$

For this step we used Runge-Kutta, but a Taylor or split-operator expansion would have been equally valid.

- Next, construct the spatial convolutions and summations that yield the generating potential, using Eqs. (40) and (46):

$$
P_{j}(\mathbf{r}, t+d t) \rightarrow Q_{j}(\mathbf{r}, t+d t) \rightarrow y(\mathbf{r}, t+d t) .
$$

- Next, construct the DKEP potential by backward derivative,

$$
u(\mathbf{r}, t+d t)=\frac{y(\mathbf{r}, t+d t)-y(\mathbf{r}, t)}{d t} .
$$

- Finally, apply the DKEP potential

$$
\Psi(\mathbf{r}, t+d t)=e^{-i u(\mathbf{r}, t+d t) d t} \Psi^{\prime}(\mathbf{r}, t+d t),
$$

- and then repeat the algorithm for the next step. 
The DKEP potential is usually quite small so that convergence did not necessitate the reduction of the time-step beyond that required for an ordinary TDOF propagation. Multiple time-step algorithms should improve the efficiency of the approach.

\section{E. Regularization}

One complication that can occur in time-dependent propagations is the possible blow-up of the equation. This will happen if the numerical value of $\operatorname{Im} \chi(q, \omega, \rho)>0$ for some value of the argument which is relevant (i.e., in the simulations below close to the plasmon resonance). Put differently, the analytical value of Im $\chi$ is never positive, but the numerical fit may lead to a small positive imaginary value. While this difficulty did not occur in our test, it could happen in future applications. To alleviate this difficulty, we could regularize the potential; the simplest way is to damp the $P_{\mathrm{j}}$ terms by adding an additional negative imaginary term, $-i \Gamma$, to the fitting frequencies:, i.e., modifying (43) to

$$
\frac{\partial P_{j}(\mathbf{r}, t)}{\partial t}=\left(-i \omega_{j}-\Gamma\right) P_{j}(\mathbf{r}, t)+\rho(\mathbf{r}, t)^{\beta_{j}} .
$$

The addition of $\Gamma$ will damp the correlation function and widen the spectrum. An alternative would be to use a frequency-based iterative scheme.

\section{SIMULATIONS}

All quantities below are in a.u. units.

\section{A. DKEP parameterization}

The DKEP was optimized for a large range of frequencies, densities, and wavevectors:

$$
0.05<\omega<0.18,0.001<\rho<0.005,0.03<q<1.8 .
$$

The resulting set of parameters for a fit with 14 terms is shown in Table I.

The RMS deviation of the real and imaginary parts of the susceptibility [from the first term on the RHS of Eq. (37)] has been, with this fit, 0.004; for comparison, the maximum of the static susceptibility is about 12 times higher at this density range.

Figure 1 shows a cut, at one value of the density and frequency, of the real and imaginary dynamic susceptibilities as a function of frequency, both the analytical and fitted expression; the agreement is generally satisfactory although the fitted susceptibility has near-singularities near $\omega=q^{2} / 2 m$ due to the structure of the TF term.

Figure 2 is similar to Fig. 1, but also includes a comparison to the susceptibility in the usual OF approach, without DKEP (i.e., purely TF $+\mathrm{VW}$ ). This usual OF susceptibility is purely real and has a very large change of values near the $\omega=q^{2} / 2 m$ range.
TABLE I. A set of parameters used to fit the DKEP potential to fit the HEG susceptibility over a large range of frequencies, densities, and wavevectors. Purely real density-exponents $\left(\alpha_{j}, \beta_{j}\right)$ were used.

\begin{tabular}{lrrrrcccr}
\hline \hline$j$ & $\operatorname{Re} d_{j}$ & $\operatorname{Im} d_{j}$ & $\operatorname{Re} \kappa_{j}$ & $\operatorname{Im} \kappa_{j}$ & $\alpha_{j}$ & $\beta_{j}$ & $\operatorname{Re} \omega_{j}$ & $\operatorname{Im} \omega_{j}$ \\
\hline 1 & 0.0364 & -0.0183 & 0.330 & -0.0634 & 0.5630 & 0.734 & -0.0023 & -0.0001 \\
2 & 0.0221 & -0.0148 & 0.489 & 1.7700 & 0.300 & 0.300 & 0.1820 & -0.0034 \\
3 & -0.0157 & 0.0420 & 0.498 & 0.1290 & 0.607 & 0.791 & -0.1480 & -0.0031 \\
4 & -0.0155 & -0.0228 & 0.406 & 0.4470 & 0.455 & 0.695 & -0.0498 & -0.0001 \\
5 & -0.0326 & 0.0070 & 0.401 & 0.2040 & 0.594 & 0.793 & -0.1230 & -0.0049 \\
6 & -0.1073 & -0.0866 & 0.400 & 1.5100 & 0.574 & 0.616 & -0.1429 & -0.0025 \\
7 & -0.0110 & -0.0078 & 0.400 & -0.0056 & 0.502 & 0.894 & 0.0940 & -0.0023 \\
8 & 0.0137 & 0.0281 & 0.410 & -0.0853 & 0.333 & 0.336 & 0.1302 & -0.0495 \\
9 & 0.0027 & -0.0057 & 0.405 & -0.0443 & 0.452 & 0.593 & 0.1779 & -0.0007 \\
10 & -0.0020 & -0.0036 & 0.484 & 0.4620 & 0.347 & 0.667 & -0.0802 & -0.0012 \\
11 & 0.0051 & -0.0158 & 0.399 & 0.0439 & 0.567 & 0.681 & 0.1152 & -0.0029 \\
12 & 0.0878 & -0.1984 & 0.400 & 1.4400 & 0.566 & 0.692 & -0.1590 & -0.0068 \\
13 & 0.0006 & 0.0009 & 0.494 & 0.3830 & 0.517 & 0.625 & -0.1571 & -0.0009 \\
14 & 0.0135 & 0.0168 & 0.394 & -0.0883 & 0.575 & 0.601 & 0.0498 & -0.0009 \\
\hline \hline
\end{tabular}

\section{B. TDOF simulations}

Next we turn to preliminary time-dependent simulation of the correlation function. As an initial test, we simulated two jellium clusters: the first, with an 8 a.u. radius associated with $\mathrm{Na}_{9}{ }^{+}$and the second, with about twice the radius, associated with $\mathrm{Na}_{65}{ }^{+}$. The maximum jellium density is 0.0042 a.u.; the density is a sigmoid function with a sharp rise. A cubic grid was used, with a spacing of 1.5 a.u and with $(16)^{3}$ points for the small simulation and $(32)^{3}$ for the large one. The smaller

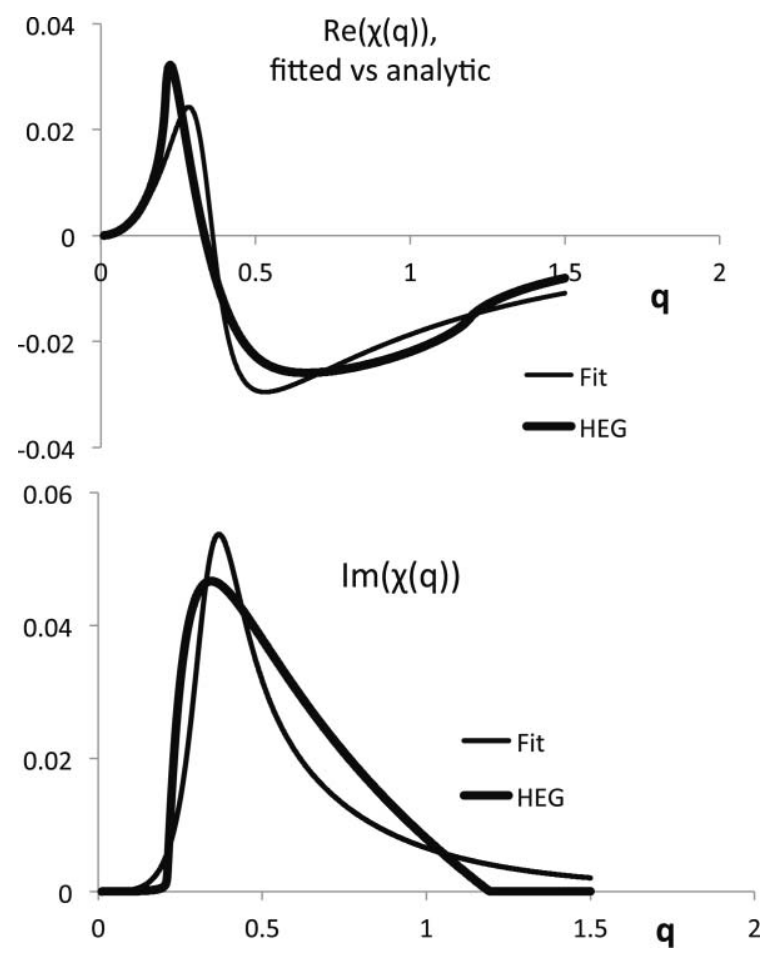

FIG. 1. Analytical vs fitted noninteracting susceptibilities for the electron gas. $\chi_{0}^{\mathrm{HEG}}(\omega, q, \rho)$ as a function of wavevector $q$, for a single value of the complex frequency $(\omega=0.125+i \cdot 0.001$ a.u. $)$ and density ( 0.004 a.u.); the numerical susceptibility was fitted within a 3D range of values, but is only shown for a single density-frequency cut. 


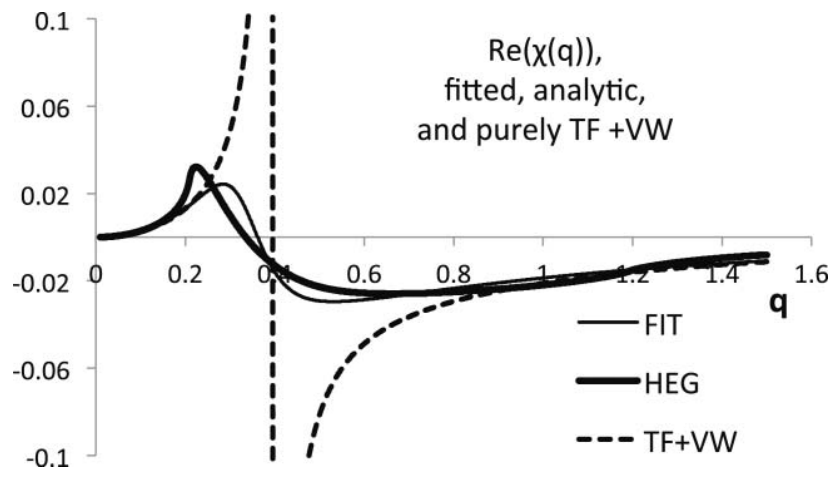

FIG. 2. Analogous to Fig. 1.a, but now including the (purely real) susceptibility of a noninteracting HEG without a dynamical kinetic energy potential (i.e., the response of a purely TF+V.W. OF Jellium). Note the different scale from Fig. 1.

cluster has been used for testing TD theories (both TDDFT and TDOFDFT), ${ }^{22}$ as well as RPA. ${ }^{35}$

Since our focus was on the dynamic response, the static simulation used a purely Thomas-Fermi and von Weizsäcker kinetic energy, without the static susceptibility term of Eq. (10); however, the Madelung Hamiltonian in Eq. (5) contains a local LDA exchange and correlation term, i.e., we used an adiabatic LDA potential in Eq. (6). Solving for the static simulation [i.e., the ground state of Eq. (5)] yields $\rho(\mathbf{r}, t=0)$.

We now added to the Madelung Hamiltonian an electric field potential, which is a delta function in time, i.e., by setting

$$
\Psi(\mathbf{r}, t=0)=\sqrt{\rho(\mathbf{r}, t=0)} e^{-v \sin \left(\frac{2 \pi z}{N_{s} d z}\right)}
$$

where we defined the number of grid points and grid spacing, and $v=0.01$ is the strength of the excitation. The Madelung function was propagated with Eqs. (5) and (47)-(51). No damping parameter $\Gamma$ was necessary.

Figure 3 shows the resulting dipoles as a function of time; for comparison, similar calculations without DKEP are included. The DKEP potential has a huge effect for the very small cluster, but as expected, its effect is less pronounced for the larger cluster, where the oscillations are much more peaked.

The DKEP has clearly two effects: first, damping of the correlation function in time, and further, a clear blue-shift of the frequency. This is demonstrated in Fig. 4, showing the imaginary part of the frequency-dependent dipole correlation function, yielding the absorption spectrum. The peak is around 0.13-0.14 a.u.; interestingly, using the standard TDDFT package Octopus, ${ }^{36}$ we have also determined the adsorption frequency to be 0.13 .

Finally, Fig. 5 shows that the DKEP is appropriate both for small and large excitations; a very large excitation of the small cluster, yielding a dipole moment of 40 a.u. for 8 electrons (i.e., an average per electron shift of 5 a.u.) still yields converging results.

These results should not be compared directly to TDDFT simulations, since no static susceptibility potential was used to fix the underlying density to fit the TDDFT static density. Put differently, the static simulations we first do in OFDFT


FIG. 3. Dipole as a function of time for $\mathrm{Na}_{9}{ }^{+}$using TDOF with a DKEP (dynamical kinetic energy potential) using a 14 term and a 28 term fit; also shown is the dipole when no DKEP potential is used (i.e., only TF+VW for the kinetic term). These simulations include an LDA exchange-correlation potential. (a) Small cluster $\left(\mathrm{Na}_{9}{ }^{+}\right)$; (b) Larger cluster $\left(\mathrm{Na}_{65}{ }^{+}\right)$.

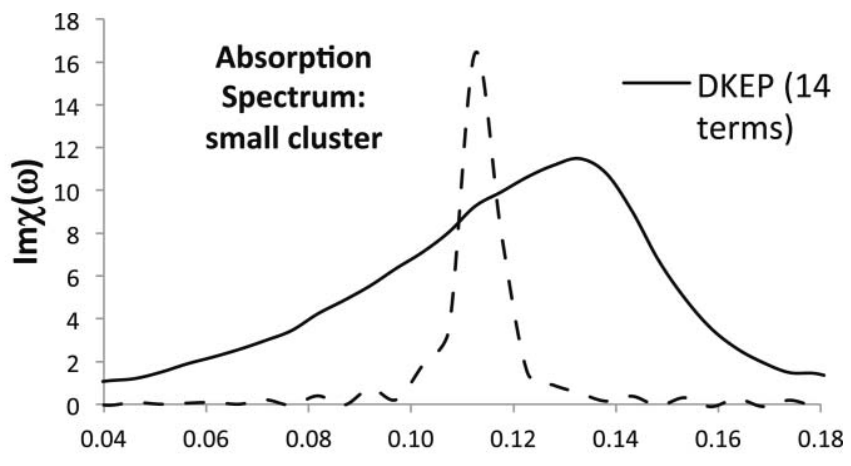

$\omega$

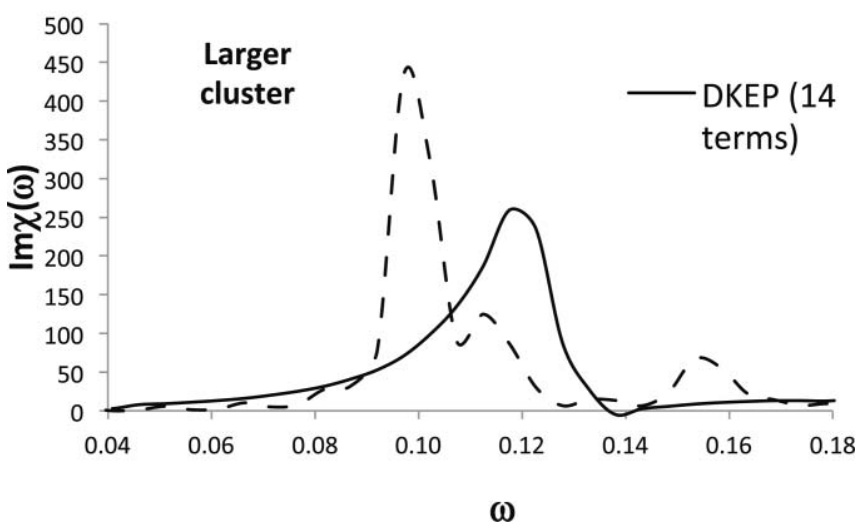

FIG. 4. $\operatorname{Im}(d(\omega))$ vs $\omega$, i.e., the absorption spectrum, for the system shown in Fig. 3; for both clusters $d(t)$ was scaled by $\exp (-0.003 \cdot t)$ prior to the Fourier transform. (a) Small cluster; the No-DKEP results scaled down by 5 for visibility; (b): larger cluster, no scaling needed. Note that the DKEP spectra get sharper for larger clusters, as expected. 


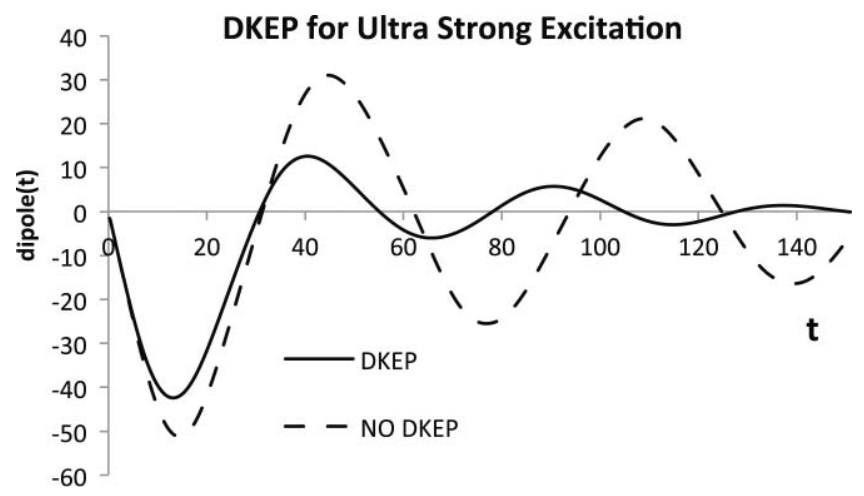

FIG. 5. DKEP for an ultrastrong excitation $(v=1$, associated with a maximum dipole of more than 6 a.u. per electron), for a small cluster. These simulation show that DKEP is useful even far away from linear response.

(before the dynamics) yield a somewhat different density than the static TDDFT results.

\section{FUTURE EXTENSIONS}

The DKEP simulations discussed here can be extended in several directions, briefly outlined below:

\section{A. Vector potentials}

The DKEP approach discussed here was based on the Madelung single-orbital propagation. However, a more general approach can be used, in which an electromagnetic potential couples to the density and currents, schematically,

$$
\begin{aligned}
& \frac{\partial \rho}{\partial t}=-\nabla \bullet \mathbf{J} \\
& \frac{\partial \mathbf{J}}{\partial t}=F(\mathbf{J}, \rho, \mathbf{A}) .
\end{aligned}
$$

Here, higher order responses will be used to construct the dynamic kinetic vector potential, $\mathbf{A}$.

\section{B. Iterative frequency-dependent approach}

We concentrated on real-time simulations. However, many applications will simply require a frequency-dependent transform rather than a real-time simulation. Such frequencydependent iterations usually require an adiabatic potential; to ensure a kinetic general potential, we simply rewrite the original propagation equation as:

$$
i \frac{d \Psi}{d t}=h_{\mathrm{eff}}\left(\rho, u, P_{j}\right) \Psi,
$$

where now the coupling is instantaneous, i.e., the propagation equations depend only on the instantaneous values of the wavefunction and of $P_{j}$ so that the linearization scheme can now work. One slight complication is the fact that the potential depends on the time derivative of $y$; recalling that the only reason for that was to make static term disappear, we define a new potential $u^{\prime}$ (to be used instead of $u$ ) that has an easy form in this context and is also devoid of a static term:

$$
\begin{aligned}
u^{\prime}(t) & =\frac{y}{\tau}-\frac{1}{\tau^{2}} \int e^{-(t-t) / \tau} y\left(t^{\prime}\right) d t^{\prime} \equiv \frac{y}{\tau}-\bar{u} \\
\frac{d \bar{u}}{d t} & =\frac{\bar{u}}{\tau}+\frac{y}{\tau^{2}}=\frac{u^{\prime}}{\tau},
\end{aligned}
$$

where $\tau$ is a short-time parameter. When coupled with Eq. (43), an effective Liouville linearized operator (" $L$ ") results,

$$
\begin{aligned}
& L\left(\begin{array}{c}
\psi_{r}(\mathbf{r}) \\
\psi_{i}(\mathbf{r}) \\
\bar{u}_{r}(\mathbf{r}) \\
\bar{u}_{i}(\mathbf{r}) \\
p_{r, j}(\mathbf{r}) \\
p_{i, j}(\mathbf{r})
\end{array}\right) \equiv\left(\begin{array}{c}
\operatorname{Re} f \\
\operatorname{Im} f \\
\operatorname{Re}\left(\frac{u^{\prime}}{\tau}\right) \\
\operatorname{Im}\left(\frac{u^{\prime}}{\tau}\right) \\
\operatorname{Re} g_{j} \\
\operatorname{Im} g_{j}
\end{array}\right) \\
& f \equiv-i \frac{1}{s}\left(h_{e f f}\left(\rho, u, P_{j}\right) \Psi-h_{e f f}\left(\rho_{0}, 0, P_{j 0}\right) \Psi_{0}\right) \quad s \rightarrow 0 \\
& g_{j}=-i \omega_{j}\left(p_{r, j}+i p_{i, j}\right)+\frac{\rho^{\beta_{j}}-\rho_{0}^{\beta_{j}}}{s},
\end{aligned}
$$

where

$$
\begin{aligned}
\Psi & =\sqrt{\rho_{0}}+s\left(\psi_{r}+i \psi_{i}\right) \\
P_{j} & =P_{0, j}+s\left(p_{r}+i p_{i}\right) \\
u & =u_{r}+i u_{i} .
\end{aligned}
$$

The Liouville operator could be then used for a frequency-dependent iterative solution, amounting to inverting $L-i \omega$ for a range of frequencies $\omega$ using iterative methods (see, e.g., Refs. 37 and 38).

An analogous approach obviously could apply for a frequency-dependent iterative solution for a general timedependent memory potential and not just DKEP.

\section{More efficient representations of the DKEP}

A possibly more efficient representation of the DKEP could be replacing Eq. (42) by

$$
\begin{aligned}
P_{j}(\mathbf{r}, t) & =\int \exp \left(-i\left(\omega_{j}-\kappa_{j} \hat{\mathbf{q}}^{2}\right)\left(t-t^{\prime}\right) \rho\left(t^{\prime}\right)^{\beta_{j}} d t^{\prime}\right. \\
& \equiv \int\left[\exp \left(-i\left(\omega_{j}-\kappa_{j} \hat{\mathbf{q}}^{2}\right)\left(t-t^{\prime}\right)\right]_{\mathbf{r}, \mathbf{r}^{\prime}} \rho\left(\mathbf{r}^{\prime}, t^{\prime}\right)^{\beta_{j}} \mathbf{d} \mathbf{r}^{\prime} d t^{\prime}\right.
\end{aligned}
$$

where $\hat{\mathbf{q}}=-i d / d r^{\prime}$, and the meaning and units of the parameter $\kappa_{j}$ is now different.

Possible advantages for this representation are twofold. First, the time evolution is now simpler:

$$
\frac{\partial P_{j}}{\partial t}=-i\left(\omega_{j}-\kappa_{j} \hat{\mathbf{q}}^{2}\right) P_{j}+\rho^{\beta_{j}},
$$

as it only involves derivatives (which could be evaluated by a few-point formulae) and not convolutions. 
Further, this presentation mixes the frequency and wavevector for each term, so that it potentially could better represent the complicated 3D landscape of the susceptibility. In fact, the Lindhard susceptibilities for the HEG are constructed by integrating over terms that are reminiscent of Eq. (58).

\section{Memory and functionals}

The DKEP as presented is a potential which is not derived from a functional; future work will aim to relate it to a functional. This task should start, from example, from aiming to satisfy the harmonic potential theorem of Dobson ${ }^{39}$; this may be achieved by casting the method in the form of an action principle on the Keldysh contour as done within orbitalTDDFT. $^{40}$

In addition, we clarify the difference between DKEP and the memory functionals in TDDFT. DKEP aims at capturing the effect of the kinetic energy for OF applications (so it is fitted to a noninteracting susceptibility); TDDFT memory functionals aim at capturing nonadiabatic exchange correlation, and should therefore be much weaker.

Finally, we note that the near singularity of the OF susceptibility when $\operatorname{Re}(\omega)=q^{2} / 2$ complicates the fit, as is evident in Fig. 1. One possible solution is to abandon the von Weizsäcker term altogether in favor of the static susceptibility potential and the DKEP; a possibly better alternative would be to replace the von Weizsäcker term by either an instantaneous term

$$
i \frac{\partial \Psi}{\partial t}=\left(f\left(q^{2}\right)+v-u\right) \Psi(t)
$$

or even a nonlocal kernel in time,

$$
i \frac{\partial \Psi}{\partial t}=\int K\left(t-t^{\prime}, \hat{\mathbf{q}}\right) \Psi\left(t^{\prime}\right) d t^{\prime}+(v-\mu) \Psi(t),
$$

where the potential depends as before on time and position, but we omitted spatial coordinates.

\section{CONCLUSIONS}

In conclusion, we outlined here the theory and have shown that DKEP is feasible with a simple and well-defined numerical representation, so that the effect of kinetic energy can, in linear response, be captured based on the HEG expressions. Further, the simple simulations demonstrate the numerical stability of DKEP.

The approach could also be extended to fit either an experimental susceptibility or a theoretically derived one for a non-HEG system. In fact, the fitting should be even simpler for experimental susceptibilities, which will not have the sharp features of the HEG susceptibility.

The DKEP potential should be a powerful tool for embedding a dynamical system described by a more accurate method such as TDDFT in a large background described by time-dependent orbital-free (TDOF) with a DKEP potential.

The type of expansion used and envisioned here should be useful for other approaches, such as memory functionals in TDDFT.
Finally, the method clearly has many possibilities, as outlined in Sec. IV. With a static susceptibility potential for the static part, as outlined, it should be possibly to include by DKEP the effects of the correct kinetic energy in a wide variety of orbital-free simulations.

\section{ACKNOWLEDGMENTS}

D.N. and G.L. are grateful for support by the NSF and ONR. Discussions with Emily Carter and Tomasz Wesolowski are gratefully acknowledged. We are also grateful to the referees for helpful comments, and in particular for supplying part of the proof used in Appendix.

\section{APPENDIX: CONNECTING TDOF TO TDDFT IN THE GENERAL CASE}

The scalar Madelung equation, i.e., the time-dependent single-particle equation for the orbital-free density, has long been used in TDOFDFT (see, e.g., Ref. 22). An (almostcomplete) proof of its formal applicability starts with TDDFT, where we know that the orbitals fulfill the TDDFT equation, Eq. (1). Left-multiplying (1) by $\phi_{j}^{*}(\mathbf{r}, t)$ and summing yields

$$
\begin{aligned}
i \sum_{j} \phi_{j}^{*} \frac{\partial \phi_{j}(\mathbf{r}, t)}{\partial t}= & -\frac{1}{2 m} \sum_{j} \phi_{j}^{*} \Delta \phi_{j}(\mathbf{r}, t) \\
& +\left(v_{\mathrm{TDDFT}}(\mathbf{r}, t)+v_{\mathrm{ext}}(\mathbf{r}, t)\right) \rho,
\end{aligned}
$$

where the sum extends only over occupied orbitals and we assume for simplicity (with no loss of generality) populations of 1 for the occupied orbitals, and 0 otherwise. The imaginary part of this equation leads to

$$
\frac{\partial \rho(\mathbf{r}, t)}{\partial t}=-\nabla \bullet \mathbf{F}(\mathbf{r}, t)
$$

where the flux is

$$
\begin{aligned}
\mathbf{F}(\mathbf{r}, t) \equiv \frac{\operatorname{Im} \sum \phi_{j}^{*} \nabla \phi_{j}(\mathbf{r}, t)}{m} & =\frac{\operatorname{Im}}{m} \sum_{j}\left(\sqrt{\rho_{j}} e^{-i \chi_{j}} \nabla \sqrt{\rho_{j}} e^{i \chi_{j}}\right) \\
& =\frac{1}{m} \sum_{j}\left(\rho_{j} \nabla \chi_{j}\right),
\end{aligned}
$$

where we defined $\phi_{j}=\sqrt{\rho_{j}} e^{i \chi_{j}}$; therefore

$$
\frac{\partial \rho(\mathbf{r}, t)}{\partial t}=-\frac{1}{m} \sum_{j}\left(\nabla \bullet\left(\rho_{j} \nabla \chi_{j}\right)\right),
$$

The TDOF equation, on the other hand, can be written as (multiplying Eq. (5) by $\Psi^{*}=|\Psi| e^{-i \chi}$, with $|\Psi|=\sqrt{\rho}$ )

$$
\begin{aligned}
& \frac{i}{2} \frac{\partial \rho}{\partial t}-\rho \frac{\partial \chi}{\partial t}=-\frac{e^{-i \chi} \sqrt{\rho} \Delta \sqrt{\rho} e^{i \chi}}{2 m}+(v-\mu) \rho \\
& =-\frac{\sqrt{\rho} \Delta \sqrt{\rho}-\rho(\nabla \chi)^{2}+i \nabla \bullet(\rho \nabla \chi)}{2 m}+(v-\mu) \rho .
\end{aligned}
$$

Therefore, in order to match the imaginary part of Eq. (A4) to (A3), we need to impose an effective Poissonlike equation for the phase (with the instantaneous electron 
density being the equivalent of the electromagnetic index of refraction):

$$
\begin{aligned}
\nabla(\rho(\mathbf{r}, t) \bullet \nabla \chi(\mathbf{r}, t)) & =2 m \nabla \bullet \mathbf{F}(\mathbf{r}, t) \\
& =\sum_{j} \nabla\left(\rho_{j}(\mathbf{r}, t) \bullet \nabla \chi_{j}(\mathbf{r}, t)\right) .
\end{aligned}
$$

We postulate without proving that this Poisson-like equation can be fulfilled, i.e., solved for $\chi$. (Note that the uniqueness of the solution to Eq. (A5) up to a constant is actually proved in exactly the same way as the analogous proof in a different context in the Runge and Gross paper (Ref. 28, the discussion after Eq. (6); here this means that in Eq. (A5) we cannot have a homogenous solution $\nabla \bullet\left(\rho(\mathbf{r}, t) \nabla \chi^{\prime}(\mathbf{r}, t)\right)=$ 0 , since if we had then we will multiply by $-\chi^{\prime}(\mathbf{r}, t)$ and integrate over space to get

$$
\begin{aligned}
0 & =-\int \chi^{\prime}(\mathbf{r}, t) \nabla\left(\rho(\mathbf{r}, t) \bullet \nabla \chi^{\prime}(\mathbf{r}, t)\right) d \mathbf{r} \\
& =\int\left(\nabla \chi^{\prime}(\mathbf{r}, t)\right)^{2} \rho(\mathbf{r}, t) d \mathbf{r},
\end{aligned}
$$

i.e., a contradiction since the density is non-negative and any practical calculation the density will be nonvanishing throughout space.)

Given then the solution to Poisson-like equation (A5), then the remaining part, i.e., the real part of Eq. (A4), is fulfilled once the DKEP potential $\left(v_{\mathrm{OF}} \equiv v-v_{\mathrm{TDDFT}}-v_{\mathrm{ext}}\right)$ is defined as

$$
\nu_{\mathrm{OF}}=-\frac{\partial \chi}{\partial t}+\frac{1}{\rho} \sum_{j} \rho_{j} \frac{\partial \chi_{j}}{\partial t}+\frac{(\sqrt{\rho}) \Delta \sqrt{\rho}-\rho(\nabla \chi)^{2}-\sum_{j}\left(\left(\sqrt{\rho_{j}}\right) \Delta \sqrt{\rho_{j}}-\rho_{j}\left(\nabla \chi_{j}\right)^{2}\right)}{2 m \rho} .
$$

Two interesting corollaries are:

- Since it is possible to numerically find the $N$-electron TDDFT orbitals for small and medium scale problems, it will be interesting to study the DKEP potential in such cases and see the deviations from the DKEP potential as defined in the main body of the paper which is developed by the fit to HEG;

- The proof here will proceed completely analogously if we use a different mass in the OF and TDDFT propagations [Eqs. (1) and (5)], except for a mass-ratio scaling factor in Eqs. (A5) and (A6), so numerically it could be useful to use a different ("effective") mass which could give a better representation of the physics of the problem.

${ }^{1}$ R. G. Parr and W. Yang, Density Functional Theory of Atoms and Molecules (Oxford University Press, Oxford, 1989).

${ }^{2}$ P. Cortona, Phys. Rev. B 44(16), 8454 (1991).

${ }^{3}$ M. Pearson, E. Smargiassi, and P. A. Madden, J. Phys.: Condens. Matter 5(19), 3221 (1993).

${ }^{4}$ A. Aguado, J. M. Lopez, J. A. Alonso, and M. J. Stott, J. Chem. Phys. 111(13), 6026 (1999).

${ }^{5}$ S. C. Watson and E. A. Carter, Comput. Phys. Commun. 128(1-2), 67 (2000).

${ }^{6}$ A. Aguado, J. M. Lopez, J. A. Alonso, and M. J. Stott, J. Phys. Chem. B 105(12), 2386 (2001).

${ }^{7}$ H. Jiang and W. T. Yang, J. Chem. Phys. 121(5), 2030 (2004).

${ }^{8}$ M. Zbiri, M. Atanasov, C. Daul, J. M. Garcia-Lastra, and T. A. Wesolowski, Chem. Phys. Lett. 397(4-6), 441 (2004).

${ }^{9}$ J. D. Chai, V. L. Lignères, G. Ho, E. A. Carter, and J. D. Weeks, Chem. Phys. Lett. 473, 263 (2009).

${ }^{10}$ T. J. Frankcombe, G. J. Kroes, N. I. Choly, and E. Kaxiras, J. Phys. Chem. B 109(34), 16554 (2005).

${ }^{11}$ V. V. Karasiev, S. B. Trickey, and F. E. Harris, J. Comput.-Aided Mater. 13(1-3), 111 (2006)

${ }^{12}$ I. V. Ovchinnikov and D. Neuhauser, J. Chem. Phys. 124(2), 024105 (2006).

${ }^{13}$ B. J. Zhou and Y. A. Wang, J. Chem. Phys. 124 (8), 081107 (2006).
${ }^{14}$ V. Gavini, K. Bhattacharya, and M. Ortiz, J. Mech. Phys. Solids 55(4), 697 (2007).

${ }^{15}$ C. J. García-Cervera, Commun. Comput. Phys. 2, 334 (2007).

${ }^{16}$ Q. Peng, X. Zhang, C. Huang, E. A. Carter, and G. Lu, Modell. Simul. Mater. Sci. 18(7), 075003 (2010).

${ }^{17}$ Q. Peng, X. Zhang, L. Hung, E. A. Carter, and G. Lu, Phys. Rev. B 78(5), 054118 (2008)

${ }^{18}$ Y. A. Wang, N. Govind, and E. A. Carter, Phys. Rev. B 60(24), 16350 (1999). Erratum: Phys. Rev. B 64, 089903 (2001).

${ }^{19}$ B. Zhou, V. L. Ligneres, and E. A. Carter, J. Chem. Phys. 122(4), 044103 (2005).

${ }^{20}$ T. A. Wesolowski and A. Warshel, J. Phys. Chem. 97(30), 8050 (1993).

${ }^{21}$ T. A. Wesolowski, Phys. Rev. A 77(1), 012504 (2008).

${ }^{22}$ A. Domps, P. G. Reinhard, and E. Suraud, Phys. Rev. Lett. 80(25), 5520 (1998).

${ }^{23}$ P. Zimmerer, M. Zimmermann, N. Grun, and W. Scheid, Comput. Phys. Commun. 63(1-3), 21 (1991).

${ }^{24}$ J. Nemeth, M. Barranco, J. Desbois, and C. Ngo, Z. Phys. A: Hadrons Nucl. 325(3), 347 (1986).

${ }^{25}$ M. Pi, M. Barranco, J. Nemeth, C. Ngo, and E. Tomasi, Phys. Lett. B 166(1), 1 (1986).

${ }^{26}$ M. Horbatsch and R. M. Dreizler, Z. Phys. A: Hadrons Nucl. 308(4), 329 (1982).

${ }^{27}$ R. Tsekov, Int. J. Theor. Phys. 48(1), 85 (2009).

${ }^{28}$ E. Runge and E. K. U. Gross, Phys. Rev. Lett. 52, 997 (1984).

${ }^{29}$ K. Andrae, P. G. Reinhard, and E. Suraud, J. Phys. B 35(20), 4203 (2002).

${ }^{30} \mathrm{~A}$. Taflove and S. Hagness, Computational Electrodynamics: The FiniteDifference Time-Domain Method, 2nd ed. (Artech House, Boston, MA, 2000).

${ }^{31}$ E. Madelung, Z. Phys. 40, 322 (1927).

${ }^{32}$ J. Lindhard, K. Dan. Vidensk. Selsk. Mat. Fys. Medd. 28, 8 (1954).

${ }^{33}$ G. H. Harris, A. J. Oconnor, and J. F. Dobson, J. Phys. C 21(1), 107 (1988).

${ }^{34}$ C. Huang and E. A. Carter, Phys. Rev. B 81(4), 045206 (2010).

${ }^{35}$ C. Yannouleas, Chem. Phys. Lett. 193(6), 587 (1992).

${ }^{36}$ M. A. L. Marques, A. Castro, G. F. Bertsch, and A. Rubio, Comput. Phys. Commun. 151(1), 60 (2003).

${ }^{37}$ D. Neuhauser and R. Baer, J. Chem. Phys. 123(20), 204105 (2005).

${ }^{38}$ R. Baer and D. Neuhauser, J. Chem. Phys. 121(20), 9803 (2004).

${ }^{39}$ J. F. Dobson, Phys. Rev. Lett. 73(16), 2244 (1994).

${ }^{40}$ Y. Kurzweil and R. Baer, Phys. Rev. B 73(7), 075413 (2006). 